\title{
Construct Validity of The Braden Scale for Predicting Pressure Sore Risk in the Long-term Care Setting: A Structural Equation Modeling Analysis of Secondary Data
}

Petra Schumacher ( $\square$ petra.schumacher@fh-krems.ac.at )

IMC University of Applied Sciences Krems

Gerhard Mueller

UMIT - Private University for Health Sciences, Medical Informatics and Technology

\section{Research Article}

Keywords: Braden Scale, construct validity, structural equation model, PLS, formative-formative model

Posted Date: January 4th, 2021

DOl: https://doi.org/10.21203/rs.3.rs-134197/v1

License: (c) (1) This work is licensed under a Creative Commons Attribution 4.0 International License.

Read Full License 


\section{Abstract}

Background: The Braden Scale for Predicting Pressure Sore Risk (Braden Scale) is based on the conceptual model by Braden and Bergstrom. The scale's psychometric properties have been tested with different methods of classical test theory; however, there is limited research on the construct validity of the scale. Therefore, this study aimed to test the construct validity of the Braden Scale with structural equation modeling (SEM) in the long-term care setting in Austria.

Methods: Secondary analysis of 311 residents' medical records were completed with SmartPLS. A formative-formative model was derived to evaluate the relationships between the measurement and latent variables of the Braden Scale. For checking the prerequisite tests for the SEM, a standard Partial Least Squares algorithm and a bootstrap procedure were used to estimate the significance of the model estimates. After calculating composite reliability of the total Braden Scale score, the overall formativeformative model was assessed for convergent and discriminant validity with the average variance extracted index (AVE) and Fornell-Larcker-Crtiterion (FLC) as well as Standardized Root Mean Square Residual fit index (SRMR).

Results: The outer weights revealed a middle positive effect for the subscale friction and shear $(\beta=$ $0.600)$; for all other subscale items, a weak positive effect $(\beta=0.261-0.430)$ on the two latent constructs Pressure and Tissue Tolerance. The inner weights showed a weak effect for the construct Tissue Tolerance $(\beta=0.423)$ and a middle effect for Pressure $(\beta=0.655)$ to the second order construct Pressure Sore Development. The latent construct was satisfactory reliable. The Braden Scale model was convergent $(\mathrm{AVE}=0.579)$ and discriminant valid $(\mathrm{FLC}$ Pressure $=0.954 ; \mathrm{FLC}$ Tissue Tolerance $=0.886)$. The model itself had a fit of SRMR $=0.146$.

Conclusions: The overall assessment of the Braden Scale show that both constructs (Pressure and Tissue Tolerance) are valid in themselves and describe the overall construct Pressure Sore Development equally valid. The separation of the items into the two latent constructs Pressure and Tissue Tolerance, might be reasonable and should be focus of further nursing research.

\section{Background}

Pressure sores or pressure ulcers (PU) are a major health concern in both acute and long-term care facilities causing pain and discomfort to the affected person. A PU is a localized damage to the skin and underlying tissue typically over bony prominences, such as sacrum, coccyx, heels, and hips regions due to pressure with or without shear (1). Risk factors include advanced age, immobility, malnutrition, vascular diseases, incontinence, and other skin conditions (2).

The prevalence varies depending on the risk population and tested setting. A hospital survey in United States acute care setting $(n=216,626)$ reported pressure injuries prevalence of $9.2 \%(n=19,893)$. The reported prevalence for the German long-term care setting was $2 \%$ to $5 \%$ and in hospitals $2 \%$ to $4 \%$ (starting at category 2). Routine surveys in the hospital sector provided heterogeneous data with 
prevalence of $0.07 \%$ to $4.37 \%$ from category 1 and $1.6 \%$ from category 2 (3). In Austria, the hospitalacquired pressure ulcer prevalence was $0.6 \%(n=3,648)$ in hospitals and $3.5 \%(n=696)$ in nursing homes for the year 2012 (4). Between 2008 and 2012, the reported prevalence in long-term care facilities in Germany were between $4.8 \%$ and $73.3 \%(n=14,798)$ depending on additional health conditions such as urinary incontinence and malnutrition (5).

Nurses play a key role in recognizing patients at risk for developing PU through their regular assessment and continuity of patient care. Standardized screening instruments, such as the Braden Scale for Predicting Pressure Sore Risk (Braden Scale) (6), may assist nurses in identifying PU risk in order to initiate preventive interventions or treat existing wounds (7).

The Braden Scale is based on the conceptual model by Braden and Bergstrom (8). Determinants and factors contributing to PU development are pressure intensity, pressure duration as well as tolerance of the skin and underlying tissues for pressure. The conceptual model underlying PU development is displayed in figure 1.

The factors mobility, activity, and sensory perception may contribute to prolonged Pressure exposure. The factors moisture, friction and shear are extrinsic factors, while the factor nutrition is an intrinsic factor. The extrinsic and intrinsic factors influence Tissue Tolerance (8). Mobility refers to the diminished ability of a person to alternate or control their body position. Activity describes a person's ability to move independently and thus, avoid prolonged pressure over bony prominences (e.g. sitting in a wheelchair or being bedridden). Sensory perception refers to a person's reduced ability to perceive an uncomfortable position and change their position accordingly. The extrinsic factor friction and shear forces are demonstrated by a person's inability to lift their body during a position change. The component of the extrinsic factor moisture refers to a person's exposure to moisture (e.g. urinary and/or fecal incontinence, perspiration). The component of the intrinsic factor nutrition comprises the intake of food in order to nourish the body and support the quality and integrity of soft tissue (8).

The Braden Scale, as displayed in figure 2, consists of six subscales to evaluate sensory perception, activity level, mobility, nutrition status, skin moisture, and friction and shear forces to assess the risk of PU. The subscales are rated independently according to the observed severity from 1 to 4 points except for the subscale friction and shear, which is ranked from 1 to 3 points (9). Hence, the total score can range from 6 to 23 points (19 to 23 points, not at risk). The lower the score, the higher the PU risk for a patient (6). Several different cut-off points have been reported. Frequently, a score of 9 or lower indicates very high risk, 10 to 12 points high risk, 13 to 14 points moderate risk, and 15 to 18 mild risk to develop a PU (7).

The Braden Scale's psychometric properties have been tested with different methods of classical test theory. The literature review revealed evidence that the Braden Scale is valid, reliable, feasible, and applicable for different populations and settings $(5-7,10)$. 
The interrater reliability and interrater agreement (Po) for the Braden Scale (11) were high in two different nursing home samples collected in 2007 and 2008 (in 2007: $\mathrm{n}=288, \mathrm{Po}=0.66, \mathrm{ICC}(1.1)=0.90, \mathrm{Cl} 95 \%$ [0.88; 0.92], standard error of the mean (SEM) $=1.00$; in 2008: $n=292, \operatorname{Po}=0.63, \operatorname{ICC}(1.1)=0.88, \mathrm{Cl} 95 \%$ [0.85; 0.91], SEM $=0.98)$. The $95 \%$ limits of agreement were -2.8 to 2.8 (2007) and -2.7 to 2.7 (2008). The most frequent measurement errors were obtained for the subscales moisture, sensory perception, and nutrition (11).

In a Meta-analysis by Park et al. (7), the pooled sensitivity and specificity were 0.72 ( $\mathrm{Cl} 95 \%$ [0.69; 0.74]) and $0.63(\mathrm{Cl} 95 \%[0.62 ; 0.64])$, and the summary receiver-operating characteristic area under the curve was $0.84(\mathrm{SE}=0.02)$. The heterogeneity indexes in sensitivity and specificity were $79.9 \%\left(X^{2}=119.57, p<\right.$ $0.001)$ and $96.4 \%\left(\chi^{2}=673.34, p<0.001\right)$, which indicates moderate to high heterogeneity across the 25 included research studies. The positive Likelihood ratio was 2.31 ( $\mathrm{Cl} 95 \%$ [1.98; 2.69]) and the negative Likelihood ratio was 0.43 ( $\mathrm{Cl} 95 \%$ [0.06; 0.51]). This resulted in an odds ratio of 6.50 (Cl $95 \%[4.64 ; 9.11])$ indicating that the odds of a diagnosed PU increase by 6.50 with the determined risk of a PU with the Braden Scale (7).

Although the Braden Scale has been tested extensively, there is limited information on the construct validity available. The convergent construct validity was tested between the mobility subscale and physical activity measured by the Motionlogger Actigraph (12). Analysis revealed that higher subscale scores are related to more physical activity $(F=31.69, p<0.001)$. Omolayo et al. (9) studied if the construct validity of the Braden moisture subscale is inversely related to the observable contact to moisture (i.e. wet or soiled briefs, frequency of brief changes). The results of the ANOVA revealed significant differences among the moisture subscale and wet observations $(F=8.78, p<0.001)$ and among the moisture subscale and frequency of brief changes $(F=4.26, p<0.0057)$. The moisture subscale was significantly correlated with frequency of wet observations (rho $=-0.233, p<0.001$ ) and soiled observations (rho $=-0.133, p<0.013$ ). This indicates a higher subscale score with reduced wet or soiled observations. The inverse but not significantly correlation between the moisture subscale and frequency of brief changes was slightly negative $(r h o=-0.105, p<0.518)(9)$.

Recently, the construct validity of the Braden Scale was tested by Chen et al. (13) in a retrospective analysis of consecutive patients $(n=2,625)$ with structural equation modeling (SEM) to reveal the relationship between the latent variable (Braden Scale total score) and measurement variables (Braden Scale subscale scores) as well as the explained variance in the measurement variables. The proposed model was maintained by $\chi^{2}{ }_{(9)}=22.854$. The comparative fit index $(C F I=0.902)$, goodness of-fit index $(\mathrm{GFI}=0.974)$, and the root mean square error of approximation (RMSEA $=0.092)$ indicated an insufficient model fit. The factor loadings of the subscales were significant; therefore, the construct validity of the Braden Scale was not confirmed in a hospital setting (13).

There is scarce research on the factor structure of the scale's variables. The literature search revealed no other study using structural equation modeling (SEM) to test the construct validity of the Braden Scale in 
the long-term care setting in Austria. Therefore, this study aimed to test the construct validity of the Braden Scale with SEM in the long-term care setting in Austria.

\section{Methods}

\section{Study Design}

The study design was a quantitative multicenter secondary analysis of data from the long-term care setting. We followed the Standardized Reporting of Secondary Data Analyses (STROSA) statement for reporting (14).

\section{Sample and Setting}

Medical records of residents with previously recorded Braden Scale total and subscale scores were included from eight long-term care facilities of the Innsbrucker Soziale Dienste (permission for access granted) in Tyrol, Austria. Residents with missing Braden Scale total and subscale scores were excluded. The sample size determination for SEM can be determined by power analysis for multiple regression models (15). Since the maximum number of independent variables (Braden subscale items) is six, the sample should include at least 130 residents to achieve a statistical power of $80 \%$ (with error probability less or equal than $5 \%$ ) to detect a coefficient of determination $\left(R^{2}\right)$ of at least $0.10 . R^{2}$ value is used to evaluate the structural model. The sample size decreases with higher $R^{2}$ values $(15,16)$.

\section{Data Collection Procedure and Materials}

Data collection started in April 2018. Medical records of the nursing home residents were checked for socio-demographic data (resident's year of birth, gender, Braden Scale total and subscale scores), extracted, and entered into a provided SPSS version 26.0 matrix solely by the procurator of quality management of the nursing homes (Innsbrucker Soziale Dienste).

The socio-demographic data of the registered nurse administrating the Braden scale at the time of the assessment (gender, year of birth, years of work experience (in categories 0-5, 6-10 years, a.s.o.) was gathered by the procurator as well.

\section{Statistical Analysis}

The socio-demographic data was analyzed in SPSS descriptively on an exploratory level, depending on the level of measurement. In addition, percentage and absolute frequencies were calculated. The Kolmogorov-Smirnov test was used to check the normal distribution of metric variables.

For the SEM, the data analyses were completed with SmartPLS (v3.3.2). Based on the conceptual schema for the study of the etiology of pressure ulcers (8), a formative-formative model was derived to evaluate the relationships between measurement and latent variables of the Braden Scale. A formative measurement model is based on the hypothesis that the latent construct (Pressure Sore Development) is 
fully formed by the composite indicators (Braden subscale items). Hypothetical constructs thus represent a linear combination of the composite indicators, which corresponds to the regression analysis approach $(16,17)$. Hereby, the latent construct Pressure Sore Development (Braden Scale total score) was understood as a second order construct or formative-formative model (18), since the Braden subscale items of the latent construct Pressure Sore Development are composed of two other first-order latent constructs, namely Pressure and Tissue Tolerance. Figure 3 displays the formative-formative model derived from the conceptual schema for the study of the etiology of pressure ulcers (8).

In a first step, the prerequisite tests for the SEM, the internal consistency ( $\mathrm{n}=6$ items; $\mathrm{n}=311$ sample) and an exploratory factor analysis (EFA), were calculated in SPSS to check the one-dimensionality of the constructs (17). In the present formative-formative model, the calculation of Cronbach's alpha values is statistically not necessary and only possible for Pressure Sore Development due to the second order model, since the other two constructs are of formative nature and do not necessarily covary (16). The threshold for Cronbach's alpha values is 0.5 , indicating a low internal consistency (with a possible value range of 0 to 1) (19). The EFA was calculated by using principal factor analysis. Data adequacy was analyzed with the Kaiser-Meyer-Olkin coefficient (KMO) and verified the existence of a moderate correlation for Pressure $(\mathrm{KMO}=0.6932)$ and a weak correlation for Tissue Tolerance $(\mathrm{KMO}=0.5626)$. The relations between the Braden subscale items to the two latent constructs Pressure and Tissue Tolerance were represented by factor loadings $(\lambda)$. Loadings indicate how strongly a factor explains a certain item with a possible range between -1 and 1 . The higher the factor loading, the closer the relationship between the subscale item and the factor (20).

A standard PLS algorithm and a bootstrap procedure was used to estimate the significance of the model estimates. The Partial Least Squares (PLS) technique was used to analyze the formative-formative model. The measurement (outer) model describes the path coefficients (outer weights, $\beta$ ) between the Braden subscales to the two latent constructs Pressure and Tissue Tolerance. The effect of the structural (inner) model describes the path coefficients (inner weights, $\beta$ ) from the two latent constructs to the second order construct Pressure Sore Development. The significance of the path coefficients and the loadings were tested with the bootstrapping method $(N=5,000)(16)$. Then, the composite reliability of Pressure Sore Development was directly calculated in SmartPLS. The reliability increases the more dissimilar the items of the factor charges on a latent construct $(16,21)$. Composite reliability considers the different loadings of the items on a construct. In terms of content, values between 0.6 and 0.7 are regarded as acceptable and values between 0.7 and 0.9 as satisfactory (16).

The next step involved the assessment of the overall formative-formative model for convergent and discriminant validity. Convergent validity is the correlation of a construct with the alternative measurement of the same construct. The index calculated is the average variance extracted (AVE), which should be greater than 0.5 to be considered valid (16). Discriminant validity refers to the fact that a latent construct really differs from other latent constructs in the model. The first way to check this was to evaluate the cross loadings between the latent factors. Overall, the cross loading of an item on its latent factor should be greater than all other cross loadings of the item on all other latent constructs together 
and thus, should belong to the latent factor to which it is theoretically assigned. Another criterion used to check discriminant validity is the Fornell-Larcker-Criterion (FLC), which compares the square root of the AVE of a latent construct, which "should be greater than its highest correlation with any other constructs" (16).

In a last step, the quality measure of the overall structural model was calculated with the Standardized Root Mean Square Residual (SRMSR) fit index, which requires a limit value of under $0.080(22,23)$.

\section{Ethical Consideration}

The study was approved by the local Research Committee for Scientific and Ethical Questions of the UMIT - Private University for Health Sciences, Medical Informatics and Technology. Residents' and registered nurses' informed consent was waived by the ethics committee due to the nature of secondary analysis of previously recorded data. Data from the residents' medical records and registered nurses were collected and anonymized solely by the procurator of quality management of the nursing homes. The transfer of anonymized data from the long-term care facility was for statistical purposes only. The authors and the statistician only received anonymized data and were blinded to residents' and registered nurses' names. Names of residents and registered nurses were also not mentioned in any scientific publications. The authors confirm that all methods were carried out in accordance with relevant guidelines and regulations. The regulations of the Data Protection Act in the current version was fulfilled by all involved study persons. These persons were subject to a statutory confidentiality obligation.

\section{Results}

\section{Socio-demographic Characteristics}

In total, 311 medical records of nursing home residents had recorded Braden Scale total and subscale scores and were extracted. The socio-demographic characteristics of the residents is summarized in table 1.

Table 1: Socio-demographic characteristics of nursing home residents $(n=311)$

\begin{tabular}{|c|c|c|c|c|c|c|c|c|}
\hline & Gender & $\mathrm{n}(\%)$ & Min & Max & MD & IQR & Mean & SD \pm \\
\hline \multirow[t]{2}{*}{ Years in age } & Female & $\begin{array}{l}235 \\
(75.6)\end{array}$ & 50 & 100 & 89.00 & $\begin{array}{l}81.00 \\
94.00\end{array}$ & 87.01 & 9.765 \\
\hline & Male & $\begin{array}{c}76 \\
(24.4)\end{array}$ & 39 & 100 & 83.50 & $\begin{array}{l}74.25 \\
90.00\end{array}$ & 82.24 & 11.651 \\
\hline \multirow{2}{*}{$\begin{array}{l}\text { Braden Scale total } \\
\text { score }\end{array}$} & Female & $\begin{array}{l}235 \\
(75.6)\end{array}$ & 6 & 23 & 18.00 & $\begin{array}{l}14.00 \\
21.00\end{array}$ & 17.03 & 4.313 \\
\hline & Male & $\begin{array}{c}76 \\
(24.4)\end{array}$ & 7 & 23 & 17.00 & $\begin{array}{l}13.00 \\
20.00\end{array}$ & 16.51 & 4.310 \\
\hline
\end{tabular}


Legend: $\mathrm{n}=$ number, $\%=$ percent, $\mathrm{Min}=$ minimum, $\mathrm{Max}=$ maximum, $\mathrm{MD}=$ median, $\mathrm{IQR}=$ interquartile range, $\mathrm{SD}=$ standard deviation.

The Kolmogorov-Smirnov test for normal distribution of the Braden Scale total score was significant for females $\left(K_{(235)}=0.146, p=0.001\right)$ and males $\left(K_{(76)}=0.120, p=0.009\right)$. Therefore, the total scores are not normal distributed by gender. The average age of the 311 residents was 85.85 (SD \pm 10.442 ) ranging from 39 to $100(M D=88.00$, IQR $=13.00$ [80.00; 93.00]) years. The average Braden Scale total score was $16.90(S D \pm 4.311)$ ranging from 6 to $23(M D=18.00, I Q R=7.00[14.00 ; 21.00])$ points.

The average age of the 35 registered nurses ( $R N$ ) administrating the Braden scale was $41.97(\mathrm{SD} \pm 8.877$ ) years ranging from 28 to $58(\mathrm{MD}=39.00, \mathrm{IQR}=16.00$ [34.00; 50.00]) years. From the 35 nurses, nine RNs had between zero to five and eleven to 15 years of working experience as an RN. Seven nurses worked as RN between six and ten years, five RNs between 16 to 20, three RNs between 21 to 25 , and two nurses over 35 years as an RN. The highest education level of seven nurses was missing $(n=28)$. Of 28 nurses, three absolved compulsory schooling, four RNs completed an apprenticeship, nine nurses completed vocational training school, one RN visited high school, seven nurses had a high school diploma or equivalent, and four RNs had a college or university degree.

\section{Statistical Analysis of Prerequisite Test Results for the SEM}

The internal consistency of the construct Pressure was highly reliable (Cronbach's alpha $=0.872$ ). The latent construct Tissue Tolerance was weakly reliable, since Cronbach's alpha was 0.573 , just above the threshold value of $0.5(21)$.

The EFA results for the construct Pressure demonstrated moderate to high loadings for the subscales mobility $(\lambda=0.927)$, activity $(\lambda=0.921)$, and sensory perception $(\lambda=0.826)$. The described weakness of the construct Tissue Tolerance was also evident in the EFA, where the construct loaded weakly on the subscale item nutrition $(\lambda=0.623)$. The subscales moisture $(\lambda=0.755)$ and friction and shear $(\lambda=0.844)$ loaded moderately on the construct Tissue Tolerance. Thus, the evaluation of the prerequisites indicate that the formative-formative model can be further analyzed in a structural equation model to test the construct validity of the Braden Scale.

\section{Statistical Analysis of Construct Validity}

The outer weights $(\beta)$ of the measurement model between the Braden subscales to the two latent constructs Pressure and Tissue Tolerance revealed a middle positive effect for the subscale friction and shear $(\beta=0.600)$; for all other subscale items, a weak positive effect $(\beta=0.261-0.430)$ on the two latent constructs. The effect of the structural model showed a weak effect for the construct Tissue Tolerance ( $\beta$ $=0.423)$ and a middle effect for Pressure $(\beta=0.655)$ to the second order construct Pressure Sore Development. Figure 3 shows the values of the measurement and structural model. The significance of the path coefficients and the loadings were tested with the bootstrapping method $(N=5,000)$. The estimation was normal distributed and highly significant for all weights and coefficients (figure 4). 


\section{Overall Assessment of the Model}

The composite reliability (CR) of the construct Pressure Sore Development demonstrated that the latent construct was satisfactory reliable (Cronbach's $a=0.845 ; C R=0.888)$.

The tested structural model of the Braden Scale was convergent valid since the average extracted variance (AVE) was calculated in this model with 0.579 and thus exceeds the required limit of 0.500 . The discriminant validity was first checked with the cross loadings between the latent factors. Since the second order construct Pressure Sore Development consists of all items, it was included in the table of cross loadings (table 2).

Table 2: Cross loadings of latent factors

\begin{tabular}{|llll|}
\hline & Pressure & Pressure Sore Development & Tissue Tolerance \\
\hline Mobility & 0.921 & 0.878 & 0.644 \\
\hline Activity & 0.923 & 0.880 & 0.654 \\
\hline Sensory Perception & 0.831 & 0.792 & 0.591 \\
\hline Moisture & 0.530 & 0.671 & 0.757 \\
\hline Friction \& Shear & 0.644 & 0.788 & 0.890 \\
\hline Nutrition & 0.355 & 0.477 & 0.539 \\
\hline
\end{tabular}

The cross loadings were assigned to the highest constructs, where they are theoretically located (table 2). Next the Fornell-Larcker-Criterion was used and showed that both latent constructs (FLC Pressure $=0.954$, FLC Tissue Tolerance $=0.886$ ) had a very high variance elucidation regarding the target construct Pressure Sore Development, which again confirms the separation into the two constructs Pressure and Tissue Tolerance.

The model itself had a fit of SRMR $=0.146$, which is above the required limit value $(<0.080)$ and can therefore be considered unacceptable, but still sufficient for random sampling.

\section{Discussion}

The Braden Scale has been extensively tested for its test-theoretic properties; however, there is scarce research on the factor structure of the scale's variables. Therefore, this study aimed to test the construct validity of the Braden Scale with structural equation modeling in the long-term care setting in Austria.

A formative-formative model (18) was derived based on the conceptual schema for the study of the etiology of pressure ulcers (8). Evaluation of the outer weights of the measurement model between the Braden subscales to the two first order latent constructs Pressure and Tissue Tolerance revealed a middle positive effect for the subscale friction and shear. For all other Braden subscale items, the outer weights 
demonstrated a weak positive effect on the two latent first order constructs. The effect of the structural model produced a weak effect for the construct Tissue Tolerance and a middle effect for the construct Pressure to the second order construct Pressure Sore Development. The model estimation was normal distributed and highly significant for all weights and coefficients. The overall assessment of the Braden Scale model estimation revealed that the latent second order construct Pressure Sore Development was satisfactory reliable. The tested measurement model of the Braden Scale was convergent valid and for the discriminant validity, the cross loadings were assigned to the highest constructs, where they are theoretically located. The FLC showed that both latent first order constructs had a very high variance elucidation regarding the target construct Pressure Sore Development and thus, confirms the separation into the two constructs Pressure and Tissue Tolerance.

No other study was identified that derived a formative-formative model to assess relationships between the latent and measurement variables of the Braden Scale. In addition, no other nursing study could be identified that specified a formative model when describing the nature of a construct. In contrast to formative models, the reflective model is characterized by the fact that the manifestations of the measurement variables are causally caused by the latent variable. This is accompanied by the assumption that changes in the latent variable lead to an effect in all the observed variables simultaneously (neglecting measurement errors) (16). Chen et al. (13) derived a non-hierarchical reflective model to map the relationships between the latent variable (pressure sore risk denoted by the Braden scale total score) and measurement variables (Braden scale subscale scores) as well as the explained variance in the measurement variables. The secondary data analysis of consecutive hospital patients ( $\mathrm{n}$ $=2,625)$ indicated an insufficient model fit and that the Braden subscales nutrition, activity, and friction and shear were not independent risk factors for Pressure Sore Development (13). The nature of a reflective model is based on classical test theory (16). So, by adopting a reflective model for the Braden Scale, the subscale items are a representation of all possible risk factors within the conceptual schema of Pressure Sore Development, and thus, correlate with each other. This indicates that the Braden subscale items should be interchangeable, and a single subscale item can be deleted without altering the construct meaning (16).

Since our study assumed a formative-formative model based on the one hand on the conceptual schema for the study of the etiology of pressure ulcers (8) and on the other hand, published formative model selection criteria (24), our results cannot be compared with those by Chen et al. (13). The adopted formative-formative nature of our model assumes that the main factors for Pressure Sore Development and thus, the cause of pressure sores, are the latent constructs Intensity and Duration of Pressure and Tissue Tolerance for Pressure (8). Contributing factors for the Intensity and Duration of Pressure are the Braden subscale items mobility, activity, and sensory perception. Contributing factors for Tissue Tolerance are the extrinsic factors and subscale items moisture and friction and shear as well as the intrinsic factor nutrition (8). Other intrinsic factors displayed in the conceptual schema are not items of the Braden scale and were as such not included in the formative-formative model for this study. To further evaluate the nature of the relationship between the latent constructs and the measurement variables and thus guide the selection of a formative model, four specific selection criteria were applied 
(24). (1) [...] changes in the measures would produce changes in the latent construct" (25); (2) each measurement item depicts a unique facet of the latent construct; (3) measurement items may or may not covary; and (4) since measurement items represent a unique facet of the latent construct, they are not likely to all have the same antecedents and effects (25). Since all four formative model selection criteria apply in our opinion to the Braden Scale and the conceptual schema (8) indicates a second order formative model, the authors agreed to evaluate a formative-formative model instead of replicating the research method by Chen et al. (13).

Several possible limitations may have influenced the results. The authors chose to evaluate the Braden Scale based on the assumption of a formative-formative model. Other researchers may have a different approach or understanding of the conceptual schema of the Braden Scale. A selection bias is possible since only the procurator of quality management collected data due to privacy policies of the nursing homes and chose, which records to include to reach the required number of at least 300 residents.

Therefore, transcription errors cannot be ruled out. The results of this study are based on secondary data analysis of data from eight nursing homes in a single state in Austria and are thus, not representative for the long-term care setting.

\section{Conclusions}

The separation of the items of the Braden Scale into two latent constructs is strongly supported by the empirical results. The individual analyses of the EFA, the SEM, and the overall assessment show that both constructs (Pressure and Tissue Tolerance) are valid in themselves and describe the overall construct equally valid. Only the Braden subscale item nutrition seems to occupy a special position, which theoretically has its cause in the distinction between extrinsic and intrinsic factors of the latent construct Tissue Tolerance as displayed in the conceptual model for the study of the etiology of pressure ulcers. Overall, all coefficients were positive, indicating that an increase in a Braden subscale item causes an increase of the first and second order latent constructs. The Braden subscale item friction and shear showed a medium strength relationship with the second order latent construct Pressure Sore Development, all other coefficients had weak correlations.

In nursing practice, the Braden Scale consists of six subscales. The subscales are rated independently according to the observed severity for a total score indicating a possible risk for PU. Regarding the presented results, a separation into two total scores, one for the subscales measuring Pressure and one for Tissue Tolerance, might be reasonable and should be focus of further nursing research.

\section{Abbreviations}

AVE Average Variance Extracted index

CFI Comparative Fit Index

Cl $95 \%$ Confidence Interval $95 \%$ 
CR Composite Reliability

EFA Exploratory Factor Analysis

FLC Fornell-Larcker-Crtiterion

IQR Interquartile Range

KMO Kaiser-Meyer-Olkin Coefficient

Max Maximum

MD Median

Min Minimum

n Number

\% Percent

p Probability

PLS Partial Least Squares

PO Interrater Agreement

PU Pressure Ulcer

$\mathbf{R}^{2}$ Coefficient of Determination

RHO Spearman's Rank Correlation

RMSEA Root Mean Square Error of Approximation

RN Registered Nurse

SD Standard Deviation

SEM Structural Equation Modeling

SRMR Standardized Root Mean Square Residual fir index

\section{Declarations}

Ethics approval and consent to participate. The study was approved by the local Research Committee for Scientific and Ethical Questions of the UMIT - Private University for Health Sciences, Medical Informatics and Technology. Residents' and registered nurses' informed consent was waived by the ethics committee 
due to the nature of secondary analysis of previously recorded data. Data from the residents' medical records and registered nurses were collected and anonymized solely by the procurator of quality management. The transfer of anonymized data from the long-term care facility was for statistical purposes only. The authors and the statistician only received anonymized data and were blinded to residents' and registered nurses' names. Names of residents and registered nurses were also not mentioned in any scientific publications. The authors confirm that all methods were carried out in accordance with relevant guidelines and regulations. The regulations of the Data Protection Act in the current version was fulfilled by all involved study persons. These persons were subject to a statutory confidentiality obligation.

Availability of data and materials. The datasets used and/or analyzed during the current study are available from the corresponding author on reasonable request. For having this data,

please contact PS. petra.schumacher@fh-krems.ac.at

Competing interest. None to declare.

Funding. Tiroler Wissenschaftsfonds (TWF). Contract number: GZ: UNI-0404-2156

Authors' contributions. Both authors designed the study; PS wrote the manuscript and GM double checked the manuscript. Data collection and anonymization was carried out solely by the procurator of quality management of the nursing homes (Innsbrucker Soziale Dienste). Statistical analysis was carried out by STATWORX ${ }^{\circledR}$. Both authors read and approved the final manuscript.

Acknowledgments. We thank the participating nursing homes and especially the procurator of quality management of the Innsbrucker Soziale Dienste who supported us during data collection.

Authors' Information. Author 1. Petra Schumacher, BScN, MScN

IMC University of Applied Sciences Krems

Department Health Sciences

Address: Piaristengasse 1, A-3500 Krems, Austria

Telephone: +43(0) $2732802-223$

Email: petra.schumacher@fh-krems.ac.at

Author 2. Gerhard Mueller, Univ.-Prof., Dr., MSc

UMIT - Private University for Health Sciences, Medical Informatics and Technology

Department of Nursing Science and Gerontology, Institute of Nursing Science 
Address: Eduard Wallnoefer-Zentrum 1, A-6060 Hall in Tyrol, Austria

Telephone: +43(0) $508648-3891$

Email: gerhard.mueller@umit.at

Corresponding author. Petra Schumacher, BScN, MScN

Email: petra.schumacher@fh-krems.ac.at

\section{References}

1. Panel NPUA, Panel EPUA, Alliance PPPI. Prevention and Treatment of Pressure Ulcers: Quick Reference Guide. Osborne Park, Australia: Cambridge Media; 2014.

2. Reddy M. Pressure ulcers. BMJ clinical evidence. 2011;2011.

3. Tomova-Simitchieva T, Akdeniz M, Blume-Peytavi U, Lahmann N, Kottner J. Die Epidemiologie des Dekubitus in Deutschland: eine systematische Übersicht. Das Gesundheitswesen. 2019;81(06):50512.

4. Halfens RJ, Meesterberends E, van Nie-Visser NC, Lohrmann C, Schönherr S, Meijers JM, et al. International prevalence measurement of care problems: results. J Adv Nurs. 2013;69(9):e5-17.

5. Lahmann NA, Tannen A, Kuntz S, Raeder K, Schmitz G, Dassen T, et al. Mobility is the key! Trends and associations of common care problems in German long-term care facilities from 2008 to 2012. Int J Nurs Stud. 2015;52(1):167-74.

6. Bergstrom N, Demuth PJ, Braden BJ. A clinical trial of the Braden Scale for Predicting Pressure Sore Risk. Nurs Clin North Am. 1987;22(2):417-28.

7. Park SH, Lee YS, Kwon YM. Predictive Validity of Pressure Ulcer Risk Assessment Tools for Elderly: A Meta-Analysis. West J Nurs Res. 2016;38(4):459-83.

8. Braden B, Bergstrom N. A conceptual schema for the study of the etiology of pressure sores. Rehabil Nurs. 1987;12(1):8-12.

9. Omolayo T, Brown K, Rapp MP, Li J, Barrett R, Horn S, et al. Construct validity of the moisture subscale of the Braden Scale for Predicting Pressure Sore Risk. Adv Skin Wound Care. 2013;26(3):122-7.

10. Bergstrom N. The Braden Scale for predicting pressure sore risk. Nurs res. 1987;36(4):205-10.

11. Kottner J, Halfens R, Dassen T. An interrater reliability study of the assessment of pressure ulcer risk using the Braden scale and the classification of pressure ulcers in a home care setting. Int $\mathrm{J}$ Nurs Stud. 2009;46(10):1307-12.

12. Powers GC, Zentner T, Nelson F, Bergstrom N. Validation of the mobility subscale of the Braden Scale for predicting pressure sore risk. Nursing Research. 2004;53(5):340-6. 
13. Chen HL, Cao YJ, Shen WQ, Zhu B. Construct Validity of the Braden Scale for Pressure Ulcer Assessment in Acute Care: A Structural Equation Modeling Approach. Ostomy Wound Manage. 2017;63(2):38-41.

14. Swart E, Schmitt J. STandardized Reporting Of Secondary data Analyses (STROSA)-Vorschlag für ein Berichtsformat für Sekundärdatenanalysen. Zeitschrift für Evidenz, Fortbildung und Qualität im Gesundheitswesen. 2014;108(8-9):511-6.

15. Cohen J. A power primer. Psychological bulletin. 1992;112(1):155.

16. Hair Jr JF, Hult GTM, Ringle C, Sarstedt M. A primer on partial least squares structural equation modeling (PLS-SEM): Sage publications; 2016.

17. Weiber R, Mühlhaus D. Strukturgleichungsmodellierung: Eine anwendungsorientierte Einführung in die Kausalanalyse mit Hilfe von AMOS, SmartPLS und SPSS: Springer-Verlag; 2014.

18. Becker J-M, Klein K, Wetzels M. Hierarchical latent variable models in PLS-SEM: guidelines for using reflective-formative type models. Long range planning. 2012;45(5-6):359-94.

19. Blanz M. Forschungsmethoden und Statistik für die Soziale Arbeit: Grundlagen und Anwendungen: Kohlhammer Verlag; 2015.

20. Bühner M. Einführung in die Test-und Fragebogenkonstruktion: Pearson Deutschland GmbH; 2011.

21. Raykov T. Scale Reliability, Cronbach's Coefficient Alpha, and Violations of Essential Tau-Equivalence with Fixed Congeneric Components. Multivariate Behav Res. 1997;32(4):329-53.

22. Hu Lt, Bentler PM. Cutoff criteria for fit indexes in covariance structure analysis: Conventional criteria versus new alternatives. Structural equation modeling: a multidisciplinary journal. 1999;6(1):1-55.

23. Sivo SA, Fan X, Witta EL, Willse JT. The search for" optimal" cutoff properties: Fit index criteria in structural equation modeling. The Journal of Experimental Education. 2006;74(3):267-88.

24. Ford LA. Selection issues of formative models. Journal of Management Development. 2017.

25. Mackenzie SB, Podsakoff PM, Jarvis CB. The problem of measurement model misspecification in behavioral and organizational research and some recommended solutions. Journal of applied psychology. 2005;90(4):710.

\section{Figures}




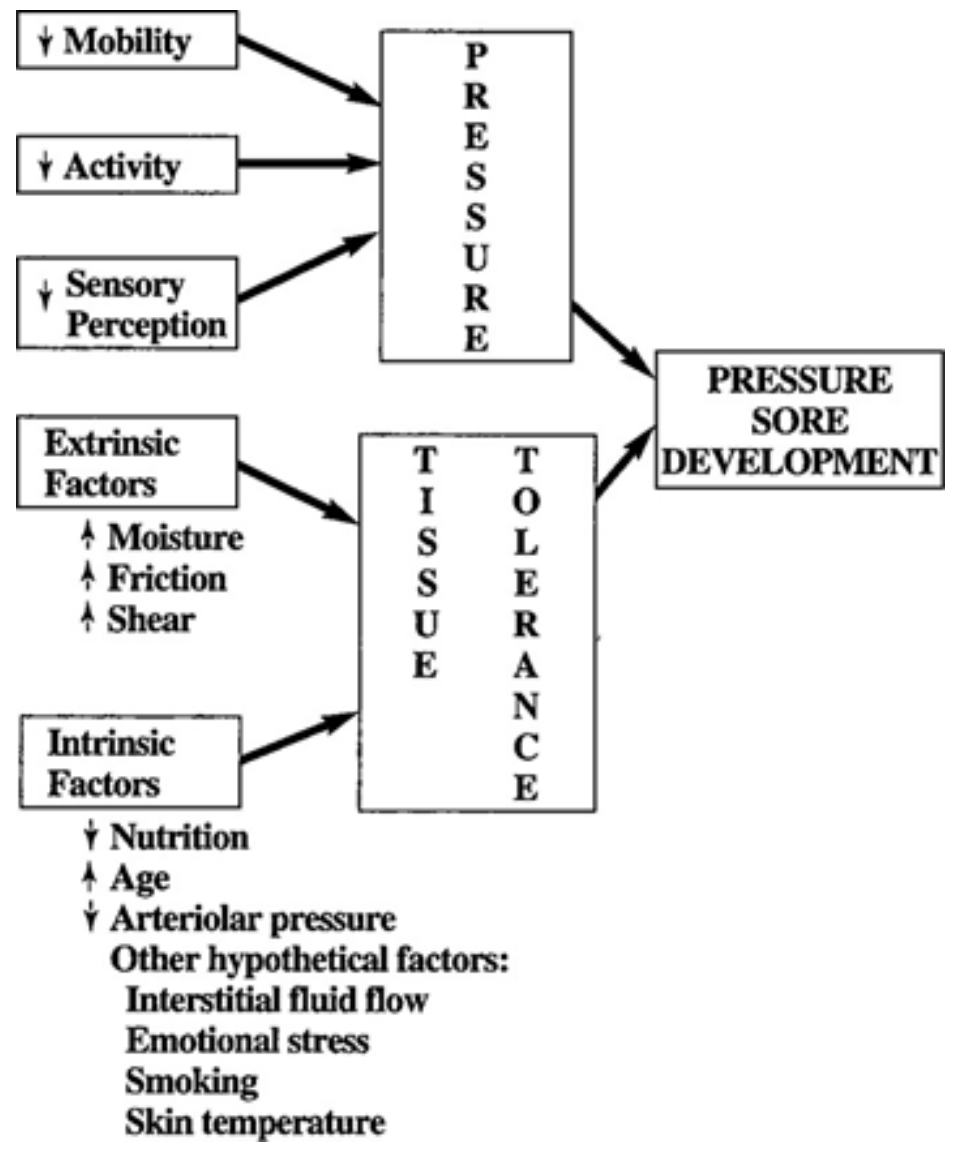

Figure 1

Conceptual model for the study of the etiology of pressure ulcers (8) 


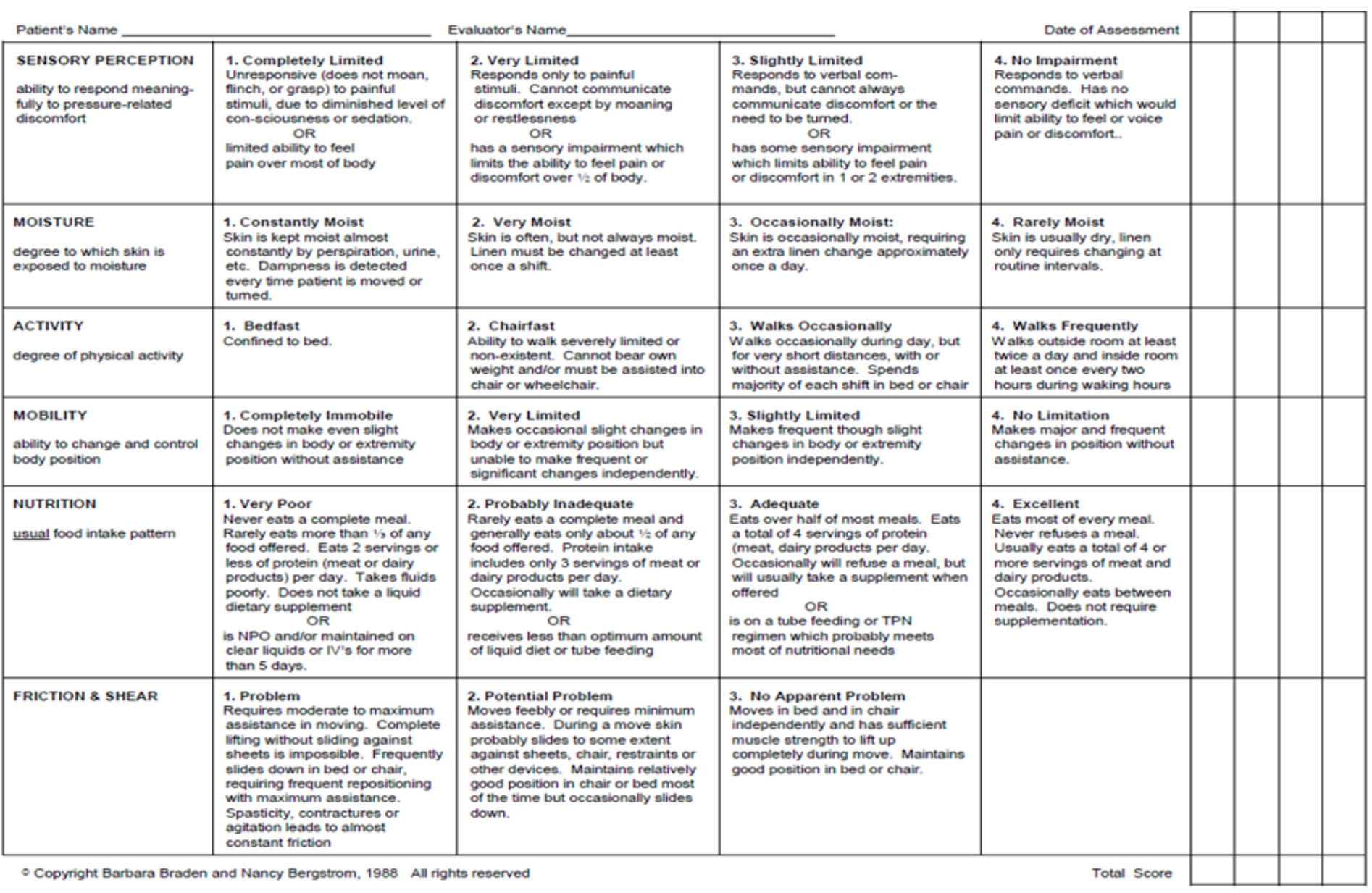

\section{Figure 2}

Braden Scale for Predicting Pressure Sore Risk

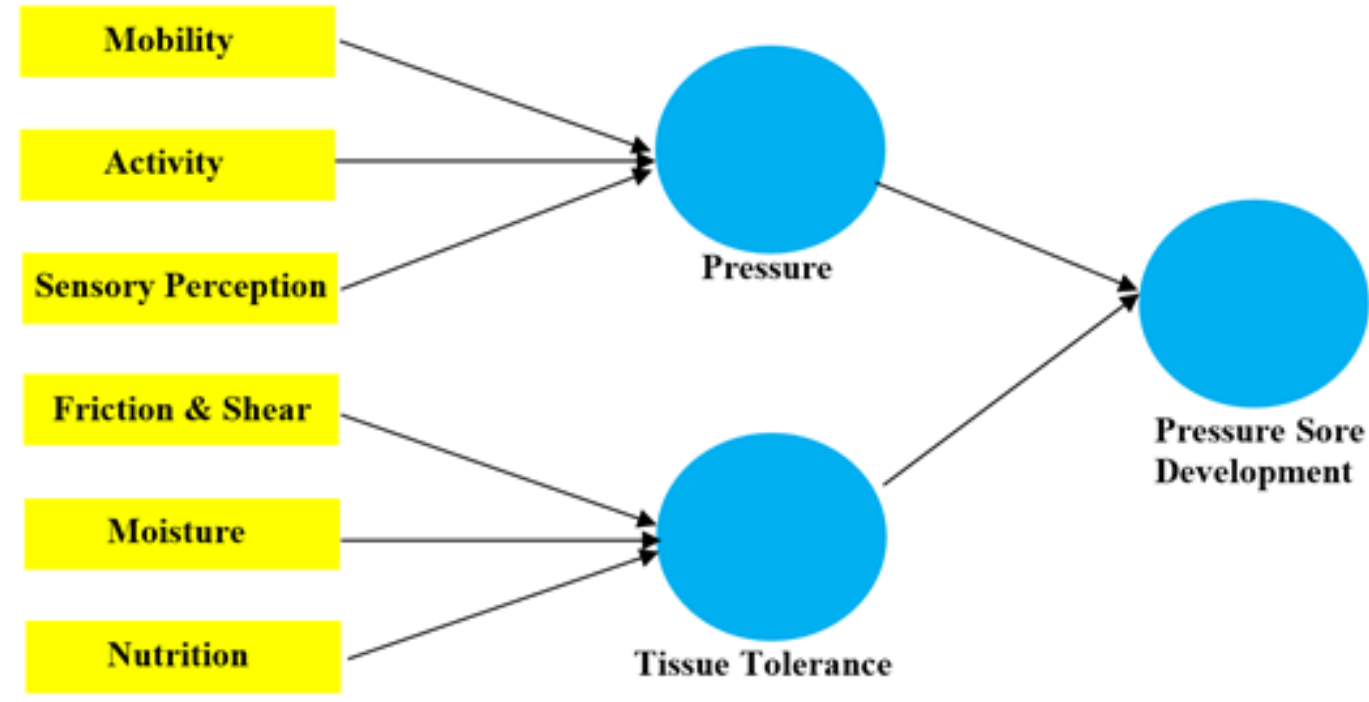

Figure 3

Formative-formative model of the Braden Scale 


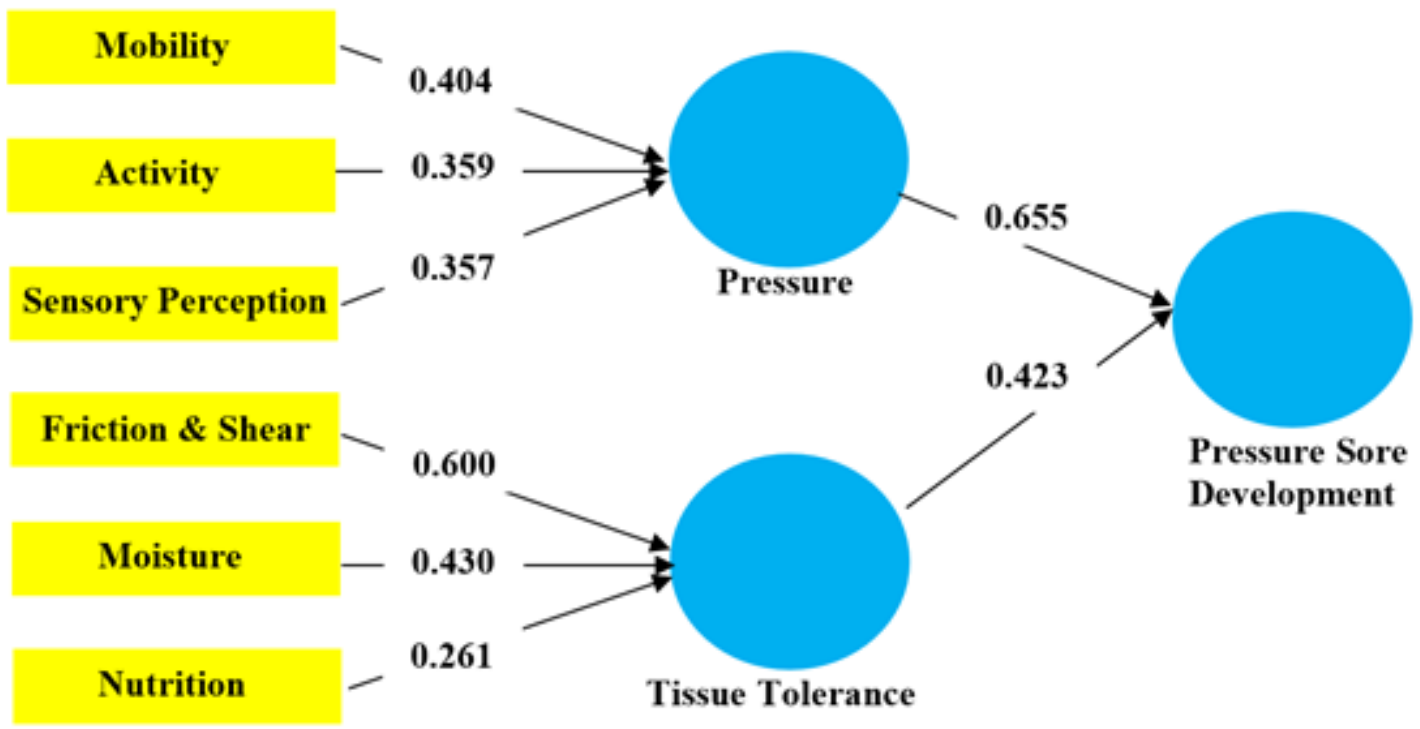

Figure 4

Path coefficients $(\beta)$ of the measurement and structural model 